\title{
Pointwise Multipliers on Spaces of Homogeneous Type in the Sense of Coifman and Weiss
}

\author{
Yanchang Han, ${ }^{1}$ Fanghui Liao, ${ }^{2}$ and Zongguang Liu ${ }^{2}$ \\ ${ }^{1}$ School of Mathematical Sciences, South China Normal University, Guangzhou 510631, China \\ ${ }^{2}$ Department of Mathematics, China University of Mining \& Technology, Beijing 100083, China
}

Correspondence should be addressed to Fanghui Liao; liaofanghui1028@163.com

Received 13 January 2014; Accepted 20 February 2014; Published 30 March 2014

Academic Editor: Changxing Miao

Copyright (c) 2014 Yanchang Han et al. This is an open access article distributed under the Creative Commons Attribution License, which permits unrestricted use, distribution, and reproduction in any medium, provided the original work is properly cited.

By applying the remarkable orthonormal basis constructed recently by Ausher and Hytönen on spaces of homogeneous type in the sense of Coifman and Weiss, pointwise multipliers of inhomogeneous Besov and Triebel-Lizorkin spaces are obtained. We make no additional assumptions on the quasi-metric or the doubling measure. Hence, the results of this paper extend earlier related results to a more general setting.

\section{Introduction}

The main purpose of this paper is to provide pointwise multipliers of inhomogeneous Besov and Triebel-Lizorkin spaces on spaces of homogeneous type in the sense of Coifman and Weiss. By a pointwise multiplier from a function space $\mathscr{A}$ into another function space $\mathscr{B}$, we meant that a function defines a bounded linear mapping from $\mathscr{A}$ into $\mathscr{B}$ by pointwise multiplication. Pointwise multipliers arise in many different areas of mathematical analysis and have many applications; for example, coefficients of differential operators and symbols of more general pseudodifferential operators may be considered as pointwise multipliers. For the theory of pointwise multipliers acting on several function spaces such as Sobolev, Besov, and Triebel-Lizorkin spaces on $\mathbb{R}^{n}$ we refer to [1]. See also [25] for more details.

It was well known that the Fourier transform is a crucial tool to study pointwise multipliers on $\mathbb{R}^{n}$. However, it was not clear how to generalize pointwise multipliers on $\mathbb{R}^{n}$ to spaces of homogeneous type introduced by Coifman and Weiss [6] because the Fourier transform is no longer available on spaces of homogenous type. To be more precise, let us first recall briefly these spaces. A quasi-metric $d$ on a set $X$ is a function $d: X \times X \rightarrow[0, \infty)$ satisfying that (i) $d(x, y)=0$ if and only if $x=y$; (ii) $d(x, y)=d(y, x)$ for all $x, y \in X$; (iii) there exists a constant $A_{0} \in[1, \infty)$ such that for all $x, y$ and $z \in X$,

$$
d(x, y) \leq A_{0}[d(x, z)+d(z, y)] .
$$

Any quasi-metric defines a topology, for which the balls

$$
B(x, r)=\{y \in X: d(y, x)<r\}
$$

for all $x \in X$ and all $r>0$ form a basis. We say that $(X, d, \mu)$ is a space of homogeneous type in sense of Coifman and Weiss if $d$ is a quasi-metric and $\mu$ is a nonnegative Borel regular measure on $X$ satisfying the doubling condition; that is, for all $x \in X, r>0$, then $0<\mu(B(x, r))<\infty$ and

$$
\mu(B(x, 2 r)) \leq C \mu(B(x, r)),
$$

where $\mu$ is assumed to be defined on a $\sigma$-algebra which contains all Borel sets and all balls $B(x, r)$ and the constant $0<C<\infty$ is independent of $x \in X$ and $r>0$. Spaces of homogenous type in the sense of Coifman and Weiss have many applications in analysis. For example, Coifman and Weiss introduced atomic Hardy space $H_{a t}^{p}$ for $p \in(0,1]$ in $[6,7]$ that proved that if $T$ is a Calderón-Zygmund singular integral operator and is bounded on $L^{2}$, then $T$ extends a bounded operator from $H^{p}$ to $L^{p}$ for suitable $p \leq 1$. However, note that the quasi-metric, in contrast to a metric, may not be Hölder regular and quasi-metric balls may not be open. For this reason, in many applications, the additional assumptions on the quasi-metric $d$ and the measure $\mu$ are required. For instance, in order to provide the maximal function characterization of the Hardy spaces $H_{a t}^{p}$ on spaces of homogenous type, Macías and Segovia in [8] showed that 
the quasi-metric $d$ can be replaced by another quasi-metric $\tilde{d}$ such that the topologies induced on $X$ by $d$ and $\widetilde{d}$ coincide, and, moreover, $\widetilde{d}$ has the following Hölder regularity: there exist positive constant $C$ and $0<\theta<1$ such that for all $0<r<\infty$ and all $x, x^{\prime}, y \in X$

$$
\begin{aligned}
& \left|\tilde{d}(x, y)-\tilde{d}\left(x^{\prime}, y\right)\right| \\
& \quad \leq C \tilde{d}\left(x, x^{\prime}\right)^{\theta}\left[\tilde{d}(x, y)+\tilde{d}\left(x^{\prime}, y\right)\right]^{1-\theta} .
\end{aligned}
$$

Furthermore, if balls $B(x, r)$ are defined by $\widetilde{d}$, that is, $B(x, r)=$ $\{y \in X: \widetilde{d}(y, x)<r\}$, then

$$
\mu(B(x, r)) \sim r .
$$

Macías and Segovia provided the maximal function characterization of the Hardy spaces $H^{p}(X)$ for $(1+\theta)^{-1}<p \leq 1$, on spaces of homogeneous type $(X, \widetilde{d}, \mu)$ with the regularity condition (4) on $\tilde{d}$ and property (5) on the measure $\mu$.

A fundamental result for these spaces $(X, \widetilde{d}, \mu)$ is the $T(b)$ theorem of David-Journé-Semmes [9], where $\widetilde{d}$ and $\mu$ satisfy (4) and (5), respectively. The crucial tool in the proof of the $T(b)$ theorem is the existence of a suitable approximation to the identity. The construction of such an approximation to the identity is due to Coifman. We would like to point out that for Coifman's construction the additional assumptions (4) on $\widetilde{d}$ and (5) on $\mu$ are crucial. Later, based on the conditions in (4) and (5), the Calderón reproducing formula, test function spaces and distributions, the Littlewood-Paley theory, and function spaces on $(X, \tilde{d}, \mu)$ were developed in [10-12].

In [13], Nagel and Stein developed the product $L^{p}(1<$ $p<\infty)$ theory in the setting of the Carnot-Carathéodory spaces formed by vector fields satisfying Hörmander's finite rank condition. The particular Carnot-Carathéodory spaces studied in [13] are spaces of homogeneous type with a smooth quasi-metric $d$ and a measure $\mu$ satisfying the conditions

$$
\mu(B(x, s r)) \sim s^{m+2} \mu(B(x, r))
$$

for $s \geq 1$ and

$$
\mu(B(x, s r)) \sim s^{4} \mu(B(x, r))
$$

for $s \leq 1$.

These conditions on the measure are weaker than property in (5) but are still stronger than the original doubling condition in (3).

Recently, pointwise multiplier theorems of Besov and Triebel-lizorkin spaces were obtained by the first author on spaces of homogeneous type with the additional assumptions (1.3) and (1.4) in [14] and with the conditions (1.3) and (1.5) in $[15,16]$.

A natural question arises: whether pointwise multipliers still hold on spaces of homogeneous type in the sense of Coifman and Weiss with only the original quasi-metric and a doubling measure?

Very recently, Auscher and Hytönen constructed an orthonormal basis with Hölder regularity and exponential decay on spaces of homogeneous type [17]. This result is remarkable since there are no additional assumptions other than those defining spaces of homogeneous type in the sense of Coifman and Weiss. Motivated by Auscher and Hytönen's orthonormal basis on spaces of homogeneous type, the purpose of the current paper is to answer the above question. More precisely, in this paper, we will provide pointwise multipliers on spaces of homogeneous type in the sense of Coifman and Weiss with the original quasi-metric $d$ and doubling measure $\mu$.

The main tool used in this paper is the orthonormal basis constructed by Auscher and Hytönen [17]. We now briefly recall the orthonormal basis constructed in [17] and inhomogeneous Besov and Triebel-Lizorkin spaces obtained in [18] on spaces of homogeneous type in the sense of Coifman and Weiss.

The orthonormal basis of $L^{2}(X)$ constructed by Auscher and Hytönen [17] is given by the following.

Theorem 1 (see [17] Theorem 7.1). Let $(X, d, \mu)$ be a space of homogeneous type in the sense of Coifman and Weiss. There exists an orthonormal basis $\psi_{\alpha}^{k}, k \in \mathbb{Z}, y_{\alpha}^{k} \in \mathcal{Y}^{k}$, of $L^{2}(X)$, having exponential decay

$$
\left|\psi_{\alpha}^{k}(x)\right| \leq \frac{C}{\sqrt{\mu\left(B\left(y_{\alpha}^{k}, \delta^{k}\right)\right)}} \exp \left(-\nu\left(\frac{d\left(y_{\alpha}^{k}, x\right)}{\delta^{k}}\right)^{a}\right),
$$

\section{Hölder-regularity}

$$
\begin{aligned}
& \left|\psi_{\alpha}^{k}(x)-\psi_{\alpha}^{k}(y)\right| \\
& \quad \leq \frac{C}{\sqrt{\mu\left(B\left(y_{\alpha}^{k}, \delta^{k}\right)\right)}}\left(\frac{d(x, y)}{\delta^{k}}\right)^{\eta} \exp \left(-v\left(\frac{d\left(y_{\alpha}^{k}, x\right)}{\delta^{k}}\right)^{a}\right)
\end{aligned}
$$

for some $\eta \in(0,1)$ and for $d(x, y) \leq \delta^{k}$, and the cancellation property

$$
\int_{X} \psi_{\alpha}^{k}(x) d \mu(x)=0, \quad k \in \mathbb{Z}, y_{\alpha}^{k} \in \mathcal{Y}^{k}
$$

Moreover,

$$
f(x)=\sum_{k \in \mathbb{Z}} \sum_{\alpha \in \mathcal{Y}^{k}}\left\langle f, \psi_{\alpha}^{k}\right\rangle \psi_{\alpha}^{k}(x)
$$

in the sense of $L^{2}(X)$.

Here $a=\left(1+2 \log 2^{A_{0}}\right)^{-1}, \delta$ is a fixed small parameter, say $\delta \leq 10^{-3} A_{0}^{-10}$, and $\nu>0$ and $C<\infty$ are constants independent of $k, \alpha, x$, and $y_{\alpha}^{k}$; see [17] for more details. In what follows, we also refer to the functions $\psi_{\alpha}^{k}$ as wavelets.

To develop function spaces such as the Hardy, Besov and Triebel-Lizorkin spaces, the key point is to introduce test function and distributions spaces. For this purpose, the following definitions were introduced in $[18,19]$. 
Definition 2. For fixed $x_{0} \in X, r>0, \gamma, \beta \in(0, \eta]$, where $\eta$ is given in Theorem 1. A function $f$ is said to be a test function of type $\left(x_{0}, r, \beta, \gamma\right)$ centered at $x_{0} \in X$ with width $r$ if $f$ satisfies the following decay and Hölder regularity properties.

(i) For all $x \in X$,

$$
|f(x)| \leq C \frac{1}{V_{r}\left(x_{0}\right)+V\left(x, x_{0}\right)}\left(\frac{r}{r+d\left(x, x_{0}\right)}\right)^{\gamma} .
$$

(ii) For all $x, y \in X$ with $d(x, y) \leq\left(1 / 2 A_{0}\right)\left(r+d\left(x, x_{0}\right)\right)$,

$$
\begin{aligned}
& |f(x)-f(y)| \\
& \quad \leq C\left(\frac{d(x, y)}{r+d\left(x, x_{0}\right)}\right)^{\beta} \frac{1}{V_{r}\left(x_{0}\right)+V\left(x, x_{0}\right)}\left(\frac{r}{r+d\left(x, x_{0}\right)}\right)^{\gamma} .
\end{aligned}
$$

If $f$ is a test function of type $\left(x_{0}, r, \beta, \gamma\right)$ centered at $x_{0} \in X$ with width $r>0$, we write $f \in \mathscr{G}\left(x_{0}, r, \beta, \gamma\right)$. The norm of $f$ on $\mathscr{G}\left(x_{0}, r, \beta, \gamma\right)$ is defined by

$$
\|f\|_{\mathscr{G}\left(x_{0}, r, \beta, \gamma\right)}=\inf \{C>0 \text { : (i) and (ii) hold }\} \text {. }
$$

We denote by $\mathscr{G}(\beta, \gamma)$ the class of all $f \in \mathscr{G}\left(x_{0}, 1, \beta, \gamma\right)$. It is easy to check that $\mathscr{G}\left(x_{1}, r, \beta, \gamma\right)=\mathscr{G}(\beta, \gamma)$ with equivalent norms for any fixed $x_{1} \in X$ and $r>0$. Furthermore, it is also easy to see that $\mathscr{G}(\beta, \gamma)$ is a Banach space with respect to the norm on $\mathscr{G}(\beta, \gamma)$.

For given $\lambda \in(0, \eta]$, let $\widetilde{\mathscr{G}}(\beta, \gamma)$ be the completion of the space $\mathscr{G}(\lambda, \lambda)$ in $\mathscr{G}(\beta, \gamma)$ with $0<\beta, \gamma \leq \lambda$. Obviously, $\widetilde{\mathscr{G}}(\lambda, \lambda)=\mathscr{G}(\lambda, \lambda)$. Moreover, $f \in \widetilde{\mathscr{G}}(\beta, \gamma)$ if and only if $f \in$ $\mathscr{G}(\beta, \gamma)$ with $0<\beta, \gamma \leq \lambda$ and there exists $\left\{f_{j}\right\}_{j \in \mathbb{N}} \subset \mathscr{G}(\lambda, \lambda)$ such that $\left\|f-f_{j}\right\|_{\mathscr{G}(\beta, \gamma)} \rightarrow 0$ as $j \rightarrow \infty$. If $f \in \widetilde{\mathscr{G}}(\beta, \gamma)$, we define $\|f\|_{\widetilde{G}(\beta, \gamma)}=\|f\|_{\mathscr{G}(\beta, \gamma)}$. Obviously, $\widetilde{\mathscr{G}}(\beta, \gamma)$ is a Banach space and we also have $\|f\|_{\widetilde{G}(\beta, \gamma)}=\lim _{j \rightarrow \infty}\left\|f_{j}\right\|_{\mathscr{G}(\beta, \gamma)}$ for the above chosen $\left\{f_{j}\right\}_{j \in \mathbb{N}}$.

We denote by $(\widetilde{\mathscr{G}}(\beta, \gamma))^{\prime}$ the dual space of $\widetilde{\mathscr{G}}(\beta, \gamma)$ consisting of all linear functional $£$ from $\widetilde{\mathscr{G}}(\beta, \gamma)$ to $\mathbb{C}$ with the property that there exists a constant $C$, for all $f \in \widetilde{\mathscr{G}}(\beta, \gamma)$,

$$
|£(f)| \leq C\|f\|_{\widetilde{G}(\beta, \gamma)} .
$$

We denote by $\langle f, h\rangle$ the natural pairing of elements $h \epsilon$ $(\widetilde{\mathscr{G}}(\beta, \gamma))^{\prime}$ and $f \in \widetilde{\mathscr{G}}(\beta, \gamma)$. Since $\widetilde{\mathscr{G}}\left(x_{1}, r, \beta, \gamma\right)=\widetilde{\mathscr{G}}(\beta, \gamma)$ with the equivalent norms for all $x_{1} \in X$ and $r>0$. Thus, for all $h \in(\widetilde{\mathscr{G}}(\beta, \gamma))^{\prime},\langle f, h\rangle$ is well defined for all $f \in$ $\widetilde{\mathscr{G}}\left(x_{0}, r, \beta, \gamma\right)$ with $x_{0} \in X$ and $r>0$.

We now give definitions of inhomogeneous Besov and Triebel-Lizorkin spaces on spaces of homogeneous type in the sense of Coifman and Weiss. Denote $P_{0}(x, y)$ $=\sum_{k \leq-1, \alpha} \psi_{\alpha}^{k}(x) \psi_{\alpha}^{k}(y)$ and $Q_{k}(x, y)=\sum_{\alpha} \psi_{\alpha}^{k}(x) \psi_{\alpha}^{k}(y)$. Let $P_{0}(f)(x)=\int P_{0}(x, y) f(y) d \mu(y)$ and $Q_{k}(f)(x)=\int Q_{k}(x$, y) $f(y) d \mu(y)$.
Definition 3 (see [18]). Let $1<p, q<\infty$ and $|s|<\eta$. The inhomogeneous Besov space $B_{p}^{s, q}(X)$ is defined by

$$
B_{p}^{s, q}(X)=\left\{f \in(\widetilde{\mathscr{G}}(\beta, \gamma))^{\prime}:\|f\|_{B_{p}^{s, q}}<\infty\right\},
$$

where

$$
\|f\|_{B_{p}^{s, q}}=\left\|P_{0}(f)\right\|_{p}+\left\{\sum_{k \geq 0}\left(\delta^{-k s}\left\|Q_{k}(f)\right\|_{p}\right)^{q}\right\}^{1 / q} .
$$

The inhomogeneous Triebel-Lizorkin space $F_{p}^{s, q}(X)$ is defined by

$$
F_{p}^{s, q}(X)=\left\{f \in(\widetilde{\mathscr{G}}(\beta, \gamma))^{\prime}:\|f\|_{F_{p}^{s, q}}<\infty\right\},
$$

where

$$
\|f\|_{F_{p}^{s, q}}=\left\|P_{0}(f)\right\|_{p}+\left\|\left\{\sum_{k \geq 0}\left(\delta^{-k s}\left|Q_{k}(f)\right|\right)^{q}\right\}^{1 / q}\right\|_{p} .
$$

We would like to point out that on $\mathbb{R}^{n}, H_{p}^{s}\left(\mathbb{R}^{n}\right)=F_{p}^{s, 2}\left(\mathbb{R}^{n}\right)$ are the Bessel-potential spaces (Lebesgue spaces, Liouville spaces). If $m=0,1,2, \ldots$ and $1<p<\infty$, then $W_{p}^{m}\left(\mathbb{R}^{n}\right)=$ $H_{p}^{m}\left(\mathbb{R}^{n}\right)=F_{p}^{m, 2}\left(\mathbb{R}^{n}\right)$ are the usual Sobolev spaces. If $0<s, 1<$ $p<\infty$ and $1 \leq q \leq \infty$, then $B_{p}^{s, q}\left(\mathbb{R}^{n}\right)$ coincides with the classical Besov spaces (Lipschitz spaces $A_{p}^{s, q}\left(\mathbb{R}^{n}\right)$ ).

In this paper, we will consider the following.

Definition 4. Suppose that $g$ is a given function on $X$. Then $g$ is called a pointwise multiplier for $B_{p}^{s, q}(X)$ if $f \rightarrow g f$ admits a bounded linear mapping from $B_{p}^{s, q}(X)$ into itself. Similarly, $g$ is called a pointwise multiplier for $F_{p}^{s, q}(X)$ if $f \rightarrow g f$ admits a bounded linear mapping from $F_{p}^{s, q}(X)$ into itself.

The main results in this paper are as follows.

Theorem 5. Let $|s|<\eta, 1<p, q<\infty$; then $g \in \mathscr{C}^{\alpha}$ is a multiplier for $B_{p}^{s, q}$ with $\alpha>|s|$. Moreover, there exists a positive constant $C$ such that

$$
\|g f\|_{B_{p}^{s, q}} \leq C\|g\|_{\mathscr{C}^{\alpha}}\|f\|_{B_{p}^{s, q}}
$$

for all $g \in \mathscr{C}^{\alpha}$ and all $f \in B_{p}^{s, q}$.

Theorem 6. Let $|s|<\eta, 1<p, q<\infty$; then $g \in \mathscr{C}^{\alpha}$ is a multiplier for $F_{p}^{s, q}$ with $\alpha>|s|$. Moreover, there exists a positive constant $C$ such that

$$
\|g f\|_{F_{p}^{s, q}} \leq C\|g\|_{\mathscr{C}^{\alpha}}\|f\|_{F_{p}^{s, q}}
$$

for all $g \in \mathscr{C}^{\alpha}$ and all $f \in F_{p}^{s, q}$.

Here, the Hölder space $\mathscr{C}^{\alpha}(X)$ is defined as the collection of $f$ such that

$$
\|f\|_{\mathscr{C}^{\alpha}}=\|f\|_{\infty}+\sup _{x \neq y} \frac{|f(x)-f(y)|}{d(x, y)^{\alpha}}<\infty .
$$


We remark that Theorems 5 and 6 were proved in [4] on $\mathbb{R}^{n}$ based on Fourier transform. As mentioned before, the Fourier transform on spaces of homogeneous type is not available and hence the idea used in [4] does not work for this more general setting. A recent work on pointwise multipliers of Besov and Triebel-Lizorkin spaces on CarnotCarathéodory spaces was developed in [14-16]. However, all results in those papers require the additional assumptions on both the quasi-metric $d$ and the measure $\mu$. Therefore, results in the present paper extend all results given in [4, 14-16].

Throughout this paper, we use $C$ to denote positive constants, whose value may change from one occurrence to the next. For the measure of ball $B(x, r)=:\{y \in X: d(x, y)<r\}$, we sometimes use the abbreviations

$$
V_{r}(x):=\mu(B(x, r)), \quad V(x, y):=V(x, d(x, y)) .
$$

A brief description of the contents of this paper is as follows. In Section 2 we prove Theorem 5. The proof of Theorem 6 will be given in Section 3 .

\section{Proof of Theorem 5}

Let $P_{0}$ and $Q_{k}$ be orthogonal projections onto $V_{0}$ and $W_{k}$ with $k \in \mathbb{N}$, respectively. The next lemma gives some estimates on kernels of operators $P_{0}$ and $Q_{k}$.

Lemma 7 (see $[17,18]$ ). Let $\eta$ be the Hölder regularity, $k \in \mathbb{N}$ and $\epsilon>0$. Suppose that $P_{0}(x, y)$ and $Q_{k}(x, y)$ are kernels of $P_{0}$ and $Q_{k}$, respectively. Then there exists a constant $C$ such that

(i)

$$
\begin{aligned}
& \left|P_{0}(x, y)\right| \leq C \frac{1}{V_{1}(x)+V(x, y)}\left(\frac{1}{1+d(x, y)}\right)^{\epsilon}, \\
& \text { (ii) for } d\left(y, y^{\prime}\right) \leq\left(1 / 2 A_{0}\right)(1+d(x, y)), \\
& \left|P_{0}(x, y)-P_{0}\left(x, y^{\prime}\right)\right| \\
& \quad \leq C\left(\frac{d\left(y, y^{\prime}\right)}{1+d(x, y)}\right)^{\eta} \frac{1}{V_{1}(x)+V(x, y)}\left(\frac{1}{1+d(x, y)}\right)^{\epsilon},
\end{aligned}
$$

(iii)

$$
\int_{X} P_{0}(x, y) d \mu(x)=1
$$

(iv)

$$
\left|Q_{k}(x, y)\right| \leq C \frac{1}{V_{\delta^{k}}(x)+V(x, y)}\left(\frac{\delta^{k}}{\delta^{k}+d(x, y)}\right)^{\epsilon},
$$

$$
\text { (v) for } d\left(y, y^{\prime}\right) \leq\left(1 / 2 A_{0}\right)\left(\delta^{k}+d(x, y)\right) \text {, }
$$

$$
\begin{aligned}
& \left|Q_{k}(x, y)-Q_{k}\left(x, y^{\prime}\right)\right| \\
& \quad \leq C\left(\frac{d\left(y, y^{\prime}\right)}{\delta^{k}+d(x, y)}\right)^{\eta} \frac{1}{V_{\delta^{k}}(x)+V(x, y)}\left(\frac{\delta^{k}}{\delta^{k}+d(x, y)}\right)^{\epsilon},
\end{aligned}
$$

(vi)

$$
\int_{X} Q_{k}(x, y) d \mu(x)=0
$$

Note that (ii), (iii), (v), and (vi) still hold with $x$ and $y$ interchanged.

The key tool used in this paper, as mentioned, is the following version of the wavelet expansion.

Lemma 8 (see [18]). Let $P_{0}$ and $\left\{Q_{k}\right\}_{k \in \mathbb{Z}_{+}}$be the same as in Definition 3. Then

$$
f=P_{0}^{2}(f)+\sum_{k \geq 0} Q_{k}^{2}(f)
$$

holds in $L^{p}$ with $1<p<\infty$.

For our purpose, we need the following lemmas.

Lemma 9 (see [18]). Let $0<\beta, \gamma<\eta$. Then (30) still holds in $\widetilde{\mathscr{G}}(\beta, \gamma)$ and $(\widetilde{\mathscr{G}}(\beta, \gamma))^{\prime}$.

Lemma 10. If $f \in \mathscr{G}(\beta, \gamma)$ with $|s|<\beta<\eta, 0<\gamma<\eta$, then $f \in B_{p}^{s, q}$ and $f \in F_{p}^{s, q}$ with $|s|<\eta, 1<p<\infty, 1<q<\infty$.

Proof. Suppose that $f \in \mathscr{G}(\beta, \gamma)$ with $|s|<\beta<\eta, 0<\gamma<\eta$. We claim that

$$
\begin{aligned}
& \left|\int_{X} P_{0}(x, y) f(x) d \mu(x)\right| \\
& \quad \leq C\|f\|_{\mathscr{G}(\beta, \gamma)} \frac{1}{V_{1}\left(x_{0}\right)+V\left(y, x_{0}\right)}\left(\frac{1}{1+d\left(x_{0}, y\right)}\right)^{\gamma}
\end{aligned}
$$

and for $k \in \mathbb{N}$

$$
\begin{aligned}
& \left|\int_{X} Q_{k}(x, y) f(x) d \mu(x)\right| \\
& \quad \lesssim \delta^{k \beta}\|f\|_{\mathscr{G}(\beta, \gamma)} \frac{1}{V_{1}\left(x_{0}\right)+V\left(y, x_{0}\right)}\left(\frac{1}{1+d\left(x_{0}, y\right)}\right)^{\gamma} .
\end{aligned}
$$

We first verify (31). By the size condition of $P_{0}$ and definition of test functions, we have

$$
\begin{aligned}
& \left|\int_{X} P_{0}(x, y) f(x) d \mu(x)\right| \\
& \leq \int_{X} \frac{C\|f\|_{\mathscr{G}(\beta, \gamma)}}{V_{1}(x)+V(x, y)}\left(\frac{1}{1+d(x, y)}\right)^{\gamma} \frac{1}{V_{1}\left(x_{0}\right)+V\left(x, x_{0}\right)} \\
& \quad \times\left(\frac{1}{1+d\left(x, x_{0}\right)}\right)^{\gamma} d \mu(x) \\
& \leq C\|f\|_{\mathscr{G}(\beta, \gamma)} \frac{1}{V_{1}\left(x_{0}\right)+V\left(y, x_{0}\right)}\left(\frac{1}{1+d\left(x_{0}, y\right)}\right)^{\gamma} .
\end{aligned}
$$


To estimate (32), by the cancellation condition on $Q_{k}(x$, $y)$, we have

$$
\begin{aligned}
& \left|\int_{X} Q_{k}(x, y) f(x) d \mu(x)\right| \\
& =\left|\int_{X} Q_{k}(x, y)[f(x)-f(y)] d \mu(x)\right| \\
& \leq \int_{W_{1}}\left|Q_{k}(x, y)\right||f(x)-f(y)| d \mu(x) \\
& \quad+\int_{W_{2}}\left|Q_{k}(x, y)\right||f(x)| d \mu(x) \\
& \quad+\int_{W_{2}}\left|Q_{k}(x, y)\right||f(y)| d \mu(x) \\
& :=R_{1}+R_{2}+R_{3},
\end{aligned}
$$

where $W_{1}=\left\{x: d(x, y) \leq\left(1 / 2 A_{0}\right)\left(1+d\left(x_{0}, y\right)\right)\right\}$ and $W_{2}=$ $X \backslash W_{1}$.

For $R_{1}$, for any $\epsilon>0$, we have

$$
\begin{aligned}
R_{1} \leq & C\|f\|_{\mathscr{G}(\beta, \gamma)} \\
& \times \int_{W_{1}} \frac{1}{V_{\delta^{k}}(x)+V(x, y)}\left(\frac{\delta^{k}}{\delta^{k}+d(x, y)}\right)^{\epsilon}\left(\frac{d(x, y)}{1+d\left(x_{0}, y\right)}\right)^{\beta} \\
& \times \frac{1}{V_{1}\left(x_{0}\right)+V\left(x_{0}, y\right)}\left(\frac{1}{1+d\left(x_{0}, y\right)}\right)^{\gamma} d \mu(x) \\
\leq & C\|f\|_{\mathscr{G}(\beta, \gamma)} \delta^{\beta k} \frac{1}{V_{1}\left(x_{0}\right)+V\left(x_{0}, y\right)}\left(\frac{1}{1+d\left(x_{0}, y\right)}\right)^{\gamma},
\end{aligned}
$$

where $\epsilon>\beta>0$.

For $R_{2}, d(x, y) \geq\left(1 / 2 A_{0}\right)\left(1+d\left(x_{0}, y\right)\right)$ implies $V(x, y) z$ $V_{1}\left(x_{0}\right)+V\left(y, x_{0}\right)$; then

$$
\begin{aligned}
R_{2} \leq & C\|f\|_{\mathscr{G}(\beta, \gamma)} \int_{W_{2}} \frac{1}{V_{\delta^{k}}(x)+V(x, y)}\left(\frac{\delta^{k}}{\delta^{k}+d(x, y)}\right)^{\epsilon} \\
& \times \frac{1}{V_{1}\left(x_{0}\right)+V\left(x_{0}, x\right)}\left(\frac{1}{1+d\left(x_{0}, x\right)}\right)^{\gamma} d \mu(x) \\
\leq & C\|f\|_{\mathscr{G}(\beta, \gamma)} \delta^{\beta k} \frac{1}{V_{1}\left(x_{0}\right)+V\left(x_{0}, y\right)}\left(\frac{1}{1+d\left(x_{0}, y\right)}\right)^{\gamma},
\end{aligned}
$$

To estimate $R_{3}$, since $d(x, y) /\left(d\left(y, x_{0}\right)+1\right) \gtrsim 1$, then

$$
\begin{aligned}
R_{3} \leq & C\|f\|_{\mathscr{G}(\beta, \gamma)} \\
& \times \int_{W_{2}} \frac{1}{V_{\delta^{k}}(x)+V(x, y)}\left(\frac{\delta^{k}}{\delta^{k}+d(x, y)}\right)^{\epsilon}\left(\frac{d(x, y)}{1+d\left(x_{0}, y\right)}\right)^{\beta} \\
& \times \frac{1}{V_{1}\left(x_{0}\right)+V\left(x_{0}, y\right)}\left(\frac{1}{1+d\left(x_{0}, y\right)}\right)^{\gamma} d \mu(x) \\
\leq & C\|f\|_{\mathscr{G}(\beta, \gamma)} \delta^{\beta k} \frac{1}{V_{1}\left(x_{0}\right)+V\left(x_{0}, y\right)}\left(\frac{1}{1+d\left(x_{0}, y\right)}\right)^{\gamma},
\end{aligned}
$$

where $\epsilon>\beta>0$. The claim is concluded.

We now return to the proof of Lemma 10 and only prove that $\mathscr{G}(\beta, \gamma) \subset B_{p}^{s, q}$ since the proof of $\mathscr{G}(\beta, \gamma) \subset F_{p}^{s, q}$ is similar. By applying (31) and (32), it follows that

$$
\begin{aligned}
\|f\|_{B_{p}^{s, q}} & =\left\|P_{0}(f)\right\|_{L^{p}}+\left\{\sum_{k=0}^{\infty}\left(\delta^{-k s}\left\|Q_{k}(f)\right\|_{L^{p}}\right)^{q}\right\}^{1 / q} \\
& \leq C\|f\|_{\mathscr{G}(\beta, \gamma)}+C\|f\|_{\mathscr{G}(\beta, \gamma)}\left\{\sum_{k=0}^{\infty}\left(\delta^{k(\beta-s)}\right)^{q}\right\}^{1 / q} \\
& \leq C\|f\|_{\mathscr{G}(\beta, \gamma)},
\end{aligned}
$$

where $\beta>s$. Thus $\mathscr{G}(\beta, \gamma) \subset B_{p}^{s, q}$.

Lemma 11. Let $g \in \mathscr{C}^{\alpha}$ and $k, l \in \mathbb{N}$. For $\epsilon>\eta$,

$$
\left|P_{0} g P_{0}(x, y)\right| \leq C\|g\|_{\mathscr{C}^{\alpha}} \frac{1}{V_{1}(x)+V(x, y)}\left(\frac{1}{1+d(x, y)}\right)^{\epsilon}
$$

$\left|Q_{k} g Q_{l}(x, y)\right| \leq C\|g\|_{\mathscr{C}^{\alpha}} \delta^{|k-l|(\alpha \wedge \eta)}$

$$
\times \frac{1}{V_{\delta^{(k \wedge l)}}(x)+V(x, y)}\left(\frac{\delta^{(k \wedge l)}}{\delta^{(k \wedge l)}+d(x, y)}\right)^{\epsilon}
$$

$$
\begin{aligned}
& \left|P_{0} g Q_{l}(x, y)\right| \\
& \quad \leq C\|g\|_{\mathscr{C}^{\alpha}} \delta^{l(\alpha \wedge \eta)} \frac{1}{V_{1}(x)+V(x, y)}\left(\frac{1}{1+d(x, y)}\right)^{\epsilon}, \\
& \quad\left|Q_{k} g P_{0}(x, y)\right| \\
& \quad \leq C\|g\|_{\mathscr{C}^{\alpha}} \delta^{k(\alpha \wedge \eta)} \frac{1}{V_{1}(x)+V(x, y)}\left(\frac{1}{1+d(x, y)}\right)^{\epsilon},
\end{aligned}
$$

where $a \wedge b$ denotes the minimum of $a$ and $b$. 
Proof. We first consider (39). By the size conditions of $P_{0}$ and the definition of Hölder space $\mathscr{C}^{\alpha}$, we have

$\left|P_{0} g P_{0}(x, y)\right| \leq C\|g\|_{\mathscr{C}^{\alpha}}$

$$
\begin{aligned}
& \times \int_{X} \frac{1}{V_{1}(x)+V(x, z)}\left(\frac{1}{1+d(z, x)}\right)^{\epsilon} \\
& \quad \times \frac{1}{V_{1}(y)+V(y, z)}\left(\frac{1}{1+d(z, y)}\right)^{\epsilon} d \mu(z) \\
& \leq C \frac{1}{V_{1}(y)+V(y, x)}\left(\frac{1}{1+d(x, y)}\right)^{\epsilon} .
\end{aligned}
$$

For (40), we only consider that the case for $l \geq k \geq 1$ and the proof for $k \geq l \geq 1$ are similar. In fact, if $l \geq k \geq 1$, we have

$$
\begin{aligned}
& \left|Q_{k} g Q_{l}(x, y)\right| \\
& =\left|\int_{X}\left[Q_{k}(x, z) g(z)-Q_{k}(x, y) g(y)\right] Q_{l}(z, y) d \mu(z)\right| \\
& \leq \int_{X}\left|Q_{k}(x, z)-Q_{k}(x, y)\right||g(z)|\left|Q_{l}(z, y)\right| d \mu(z) \\
& \quad+\int_{X}\left|Q_{k}(x, y)\right||g(z)-g(y)|\left|Q_{l}(z, y)\right| d \mu(z) \\
& =: L_{1}+L_{2} .
\end{aligned}
$$

We estimate $L_{1}$ by further splitting it into

$$
\begin{aligned}
L_{1}= & \int_{W_{1}}\left|Q_{k}(x, z)-Q_{k}(x, y)\right||g(z)|\left|Q_{l}(z, y)\right| d \mu(z) \\
& +\int_{W_{2}}\left|Q_{k}(x, z)\right||g(z)|\left|Q_{l}(z, y)\right| d \mu(z) \\
& +\int_{W_{2}}\left|Q_{k}(x, y)\right||g(z)|\left|Q_{l}(z, y)\right| d \mu(z) \\
= & : L_{11}+L_{12}+L_{13},
\end{aligned}
$$

where $W_{1}=\left\{z \in X: d(z, y) \leq\left(1 / 2 A_{0}\right)\left(\delta^{k}+d(x, y)\right)\right\}$ and $W_{2}=X \backslash W_{1}$.

For $L_{11}$, for any $\epsilon>0$, we have

$L_{11} \leq C\|g\|_{\mathscr{C}^{\alpha}}$

$$
\begin{aligned}
& \times \int_{W_{1}}\left(\frac{d(z, y)}{\delta^{k}+d(x, y)}\right)^{\eta} \frac{1}{V_{\delta^{k}}(x)+V(x, y)}\left(\frac{\delta^{k}}{\delta^{k}+d(x, y)}\right)^{\epsilon} \\
& \times \frac{1}{V_{\delta^{l}}(y)+V(y, z)}\left(\frac{\delta^{l}}{\delta^{l}+d(z, y)}\right)^{\epsilon} d \mu(z)
\end{aligned}
$$

$$
\begin{aligned}
& \leq C\|g\|_{\mathscr{C}^{\alpha}} \delta^{(l-k) \eta} \frac{1}{V_{\delta^{k}}(x)+V(x, y)}\left(\frac{\delta^{k}}{\delta^{k}+d(x, y)}\right)^{\epsilon} \\
& \times \int_{X} \frac{1}{V_{\delta^{l}}(y)+V(y, z)}\left(\frac{\delta^{l}}{\delta^{l}+d(z, y)}\right)^{\epsilon-\eta} d \mu(z) \\
& \leq C\|g\|_{\mathscr{C}^{\alpha}} \delta^{(l-k) \eta} \frac{1}{V_{\delta^{k}}(x)+V(x, y)}\left(\frac{\delta^{k}}{\delta^{k}+d(x, y)}\right)^{\epsilon},
\end{aligned}
$$

where $\epsilon>\eta$.

For $L_{12}$, note that $\left(1 / 2 A_{0}\right)\left(\delta^{k}+d(x, y)\right) \leq d(z, y)$ implies that $V_{\delta^{k}}(y)+V(x, y) \lesssim V(z, y)$; then we have

$$
\begin{aligned}
L_{12} \leq & C\|g\|_{\mathscr{C}^{\alpha}} \frac{1}{V_{\delta^{k}}(x)+V(x, y)}\left(\frac{\delta^{l}}{\delta^{k}+d(x, y)}\right)^{\epsilon} \\
& \times \int_{X} \frac{1}{V_{\delta^{k}}(x)+V(y, x)}\left(\frac{\delta^{k}}{\delta^{k}+d(z, x)}\right)^{\epsilon} d \mu(z) \\
\leq & C\|g\|_{\mathscr{C}^{\alpha}} \delta^{(l-k) \eta} \frac{1}{V_{\delta^{k}}(x)+V(x, y)}\left(\frac{\delta^{k}}{\delta^{k}+d(x, y)}\right)^{\epsilon},
\end{aligned}
$$

where $\epsilon>\eta$.

For $L_{13}$, we have

$$
\begin{aligned}
L_{13} \leq & C\|g\|_{\mathscr{C}^{\alpha}} \frac{1}{V_{\delta^{k}}(x)+V(x, y)}\left(\frac{\delta^{k}}{\delta^{k}+d(x, y)}\right)^{\epsilon} \\
& \times \int_{W_{2}} \frac{1}{V_{\delta^{l}}(y)+V(y, z)}\left(\frac{\delta^{l}}{\delta^{l}+d(z, y)}\right)^{\epsilon} d \mu(z) .
\end{aligned}
$$

Denoting $t=\left(1 / 2 A_{0}\right)\left(\delta^{k}+d(x, y)\right)$, then

$$
\begin{gathered}
\int_{W_{2}} \frac{1}{V_{\delta^{l}}(y)+V(y, z)}\left(\frac{\delta^{l}}{\delta^{l}+d(z, y)}\right)^{\epsilon} d \mu(z) \\
=\sum_{j=0}^{\infty} \int_{2^{j} t<d(z, y) \leq 2^{j+1} t} \frac{1}{V_{\delta^{l}}(y)+V(y, z)} \\
\quad \times\left(\frac{\delta^{l}}{\delta^{l}+d(z, y)}\right)^{\epsilon} d \mu(z) \\
\leq \sum_{j=0}^{\infty}\left(\frac{\delta^{l}}{2^{j} t}\right)^{\epsilon} \frac{1}{V_{2^{j} t}(y)} \int_{d(z, y) \leq 2^{j+1} t} d \mu(z) \\
\leq C \sum_{j=0}^{\infty}\left(\frac{\delta^{l}}{\delta^{k}}\right)^{\epsilon} \frac{V_{2^{j+1} t}(y)}{V_{2^{j} t}(y)} \leq C \delta^{(l-k) \epsilon} .
\end{gathered}
$$

Thus

$$
L_{13} \leq C\|g\|_{\mathscr{C}^{\alpha}} \delta^{(l-k) \eta} \frac{1}{V_{\delta^{k}}(x)+V(x, y)}\left(\frac{\delta^{k}}{\delta^{k}+d(x, y)}\right)^{\epsilon},
$$


where $\epsilon>\eta$ and we obtain

$$
L_{1} \leq C\|g\|_{\mathscr{C}^{\alpha}} \delta^{(l-k) \eta} \frac{1}{V_{\delta^{k}}(x)+V(x, y)}\left(\frac{\delta^{k}}{\delta^{k}+d(x, y)}\right)^{\epsilon} .
$$

We now return to verify $L_{2}$. By the size condition of $Q_{k}$, $Q_{l}$ and the definition of $\mathscr{C}^{\alpha}$, we have

$$
\begin{aligned}
L_{2} \leq & C\|g\|_{\mathscr{C}^{\alpha}} \frac{1}{V_{\delta^{k}}(x)+V(x, y)}\left(\frac{\delta^{k}}{\delta^{k}+d(x, y)}\right)^{\epsilon} \\
& \times \int_{X} d(z, y)^{\alpha} \frac{1}{V_{\delta^{l}}(y)+V(z, y)}\left(\frac{\delta^{l}}{\delta^{l}+d(z, y)}\right)^{\epsilon} d \mu(x) \\
\leq & C\|g\|_{\mathscr{C}^{\alpha}} \delta^{l \alpha} \frac{1}{V_{\delta^{k}}(x)+V(x, y)}\left(\frac{\delta^{k}}{\delta^{k}+d(x, y)}\right)^{\epsilon},
\end{aligned}
$$

where $\epsilon>\alpha$. Then

$$
\begin{aligned}
& L_{1}+L_{2} \\
& \quad \leq C\|g\|_{\mathscr{C}^{\alpha}} \delta^{(l-k)(\alpha \wedge \eta)} \frac{1}{V_{\delta^{k}}(x)+V(x, y)}\left(\frac{\delta^{k}}{\delta^{k}+d(x, y)}\right)^{\epsilon},
\end{aligned}
$$

where $l \geq k$.

The proofs of (41) and (42) are similar to the proof of (40) and we omit the details.

We are now ready to prove Theorem 5 .

Proof of Theorem 5. We first show Theorem 5 for the special case; that is, if $f \in \mathscr{G}(\beta, \gamma)$ with $|s|<\beta<\eta, 0<\gamma<\eta$ and $g \in \mathscr{C}^{\alpha}$ with $|s|<\alpha$, then

$$
\|f g\|_{B_{p}^{s q q}} \leq C\|g\|_{\mathscr{G}^{\alpha}}\|f\|_{B_{p}^{s q q}}
$$

To verify (54), we write

$$
\begin{aligned}
\|f g\|_{B_{p}^{s, q}} & =\left\|P_{0}(g f)\right\|_{L^{p}}+\left\{\sum_{k=0}^{\infty}\left(\delta^{-k s}\left\|Q_{k}(g f)\right\|_{L^{p}}\right)^{q}\right\}^{1 / q} \\
& =: Y_{1}+Y_{2} .
\end{aligned}
$$

By the wavelet expansion, Hölder's inequality, and the estimates in (39) and (41), we obtain

$$
\begin{aligned}
Y_{1} \leq & \left\|P_{0} g\left(P_{0}^{2}(f)+\sum_{l=0}^{\infty} Q_{l}^{2}(f)\right)\right\|_{L^{p}} \\
\leq & \left\|P_{0} g P_{0} P_{0}(f)\right\|_{L^{p}}+\left\|\sum_{l=0}^{\infty} P_{0} g Q_{l} Q_{l}(f)\right\|_{L^{p}} \\
\leq & C\|g\|_{\mathscr{C}^{\alpha}}\left\|E_{0}(f)\right\|_{L^{p}}+C\|g\|_{\mathscr{C}^{\alpha}} \\
& \times \sum_{l=0}^{\infty} \delta^{l(\alpha \wedge \eta+s)} \delta^{-l s}\left\|D_{l}(f)\right\|_{L^{p}} \\
\leq & C\|g\|_{\mathscr{C}^{\alpha}}\left\|E_{0}(f)\right\|_{L^{p}}+C\left\|_{g}\right\|_{\mathscr{C}^{\alpha}} \\
& \times\left\{\sum_{l=0}^{\infty}\left(\delta^{-l s}\left\|D_{l}(f)\right\|_{L^{p}}\right)^{q}\right\}^{1 / q} \\
\leq & C\|g\|_{\mathscr{C}^{\alpha}}\|f\|_{B_{p}^{s, q}},
\end{aligned}
$$

where we use the fact that $(\alpha \wedge \eta)+s>0$ and in the third inequality, by (39) and (41), we use the estimates $\int\left|P_{0} g P_{0}(x, y)\right| d \mu(x) \leq C\|g\|_{\mathscr{C}^{\alpha}}, \int\left|P_{0} g P_{0}(x, y)\right| d \mu(y) \leq$ $C\|g\|_{\mathscr{C}^{\alpha}}, \int\left|P_{0} g Q_{l}(x, y)\right| d \mu(x) \leq C\|g\|_{\mathscr{C}^{\alpha}} \delta^{l(\alpha \wedge \eta)}$ and $\int\left|P_{0} g Q_{l}(x, y)\right| d \mu(y) \leq C\|g\|_{\mathscr{C}^{\alpha}} \delta^{l(\alpha \wedge \eta)}$.

For $Y_{2}$, instead of using (40) and (42), we have

$$
Y_{2} \leq\left\{\sum_{k=0}^{\infty}\left(\delta^{-k s}\left\|Q_{k}\left(g\left(P_{0}^{2}(f)+\sum_{l=0}^{\infty} Q_{l}^{2}(f)\right)\right)\right\|_{L^{p}}\right)^{q}\right\}^{1 / q}
$$

$$
\begin{aligned}
& \leq C\left\{\sum_{k=0}^{\infty}\left(\delta^{-k s} \delta^{k(\alpha \wedge \eta)}\left\|P_{0}(f)\right\|_{L^{p}}\right)^{q}\right\}^{1 / q} \\
& +C\left\{\sum_{k=0}^{\infty}\left(\delta^{-k s}\left\|Q_{k} g Q_{l} Q_{l}(f)\right\|_{L^{p}}\right)^{q}\right\}^{1 / q}
\end{aligned}
$$

$\leq C\|g\|_{\mathscr{C}^{\alpha}}\left\|P_{0}(f)\right\|_{L^{p}}$

$$
+C\left\{\sum_{k=0}^{\infty}\left(\sum_{l=0}^{\infty} \delta^{-k s} \delta^{|k-l|(\alpha \wedge \eta)}\|g\|_{\mathscr{C}^{\alpha}}\left\|Q_{l}(f)\right\|_{L^{p}}\right)^{q}\right\}^{1 / q}
$$

$\leq C\|g\|_{\mathscr{C}^{\alpha}}\left\|P_{0}(f)\right\|_{L^{p}}$

$+C\|g\|_{\mathscr{C}^{\alpha}}\left\{\sum_{l=0}^{\infty} \sum_{k=0}^{\infty} \delta^{(l-k) s} \delta^{|k-l|(\alpha \wedge \eta)}\left(\delta^{-l s}\left\|Q_{l}(f)\right\|_{L^{p}}\right)^{q}\right\}^{1 / q}$

$\leq C\|g\|_{\mathscr{C}^{\alpha}}\left\|P_{0}(f)\right\|_{L^{p}}$

$$
+C\|g\|_{\mathscr{C}^{\alpha}}\left\{\sum_{l=0}^{\infty}\left(\delta^{-l s}\left\|Q_{l}(f)\right\|_{L^{p}}\right)^{q}\right\}^{1 / q}
$$

$\leq C\|g\|_{\mathscr{C}^{\alpha}}\|f\|_{B_{p}^{s, q}}$ 
where $|s|<\eta$ and $|s|<\alpha$. Thus,

$$
Y_{1}+Y_{2} \leq C\|g\|_{\mathscr{C}^{\alpha}}\|f\|_{B_{p}^{s, q}}
$$

This completes the proof of (54).

To show Theorem 5 for $f \in B_{p}^{s, q}$, note that if $f \in B_{p}^{s, q}$, in general, $f$ could be a distribution and the multiplication of $g f$ is not well defined even for $g \in \mathscr{C}^{\alpha}$. For this purpose, we make the following observation: for any $f \in B_{p}^{s, q}$ with $1<p$, $q<\infty,|s|<\eta$ and $g \in \mathscr{C}^{\alpha}$ with $\alpha>|s|$, there exists a sequence $\left\{f_{n}\right\}_{n \in \mathbb{N}}$ such that $f_{j} \in \mathscr{G}(\lambda, \lambda)$ with $0<\lambda<\eta$, $\left\|f_{n}\right\|_{B_{p}^{s, q}} \leqslant\|f\|_{B_{p}^{s, q}}$ and $\lim _{n \rightarrow \infty}\left\langle g f_{n}, h\right\rangle$ converges for any $h \epsilon$ $\widetilde{\mathscr{G}}(\beta, \gamma)$ with $\beta, \gamma$ satisfying $|s|<\beta<\eta, 0<\gamma<\eta$. Indeed, for any $f \in B_{p}^{s, q}$ with $1<p, q<\infty$, $|s|<\eta$, set

$$
f_{n}=\sum_{k=-1}^{n} B_{k}^{2}(f),
$$

where $B_{-1}=P_{0}, B_{k}=Q_{k}$ for $k \in \mathbb{Z}_{+}$. By the Proposition 4.4 of [18], $f_{n} \in \widetilde{\mathscr{G}}(\lambda, \lambda)$, and $\left\|f_{n}\right\|_{B_{p}^{s, q}} \lesssim\|f\|_{B_{p}^{s, q}}$. Now we prove that $\lim _{n \rightarrow \infty}\left\langle g f_{n}, h\right\rangle$ converges for any $h \in \widetilde{\mathscr{G}}(\beta, \gamma)$ with $\beta, \gamma$ satisfying $|s|<\beta<\eta, 0<\gamma<\eta$. To do this, for $n, m \in \mathbb{N}_{+}$, $m<n$, by duality in [18] and the estimate in (54), we have

$$
\begin{aligned}
\left|\left\langle f_{n}-f_{m}, g h\right\rangle\right| & \leq\left\|f_{n}-f_{m}\right\|_{B_{p}^{s, q}}\|g h\|_{B_{p \prime^{-s, q^{\prime}}}} \\
& \leq C\|g\|_{\mathscr{C}^{\alpha}}\left\|f_{n}-f_{m}\right\|_{B_{p}^{s, q}}\|h\|_{B_{p \prime^{-s, \prime^{\prime}}} .}
\end{aligned}
$$

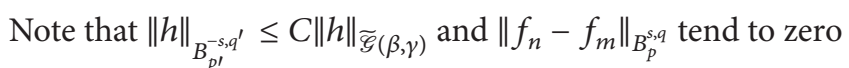
as $n, m$ tend to infinity. This implies that $\left|\left\langle f_{n}-f_{m}, g h\right\rangle\right| \rightarrow 0$ as $n, m \rightarrow \infty$ with $1<p, q<\infty,|s|<\eta$.

Now for any $g \in \mathscr{C}^{\alpha}$ with $|s|<\alpha<\eta$ and $f \in B_{p}^{s, q}$ with $1<$ $p, q<\infty,|s|<\eta$, by the above observation, $\lim _{n \rightarrow \infty}\left\langle g f_{n}, h\right\rangle$ exists. Therefore, we define

$$
\langle g f, h\rangle=\lim _{n \rightarrow \infty}\left\langle g f_{n}, h\right\rangle
$$

for $h \in \widetilde{\mathscr{G}}(\beta, \gamma)$ with $\beta, \gamma$ satisfying $|s|<\beta<\eta, 0<\gamma<\eta$. It is easy to see that limit is independent of the choice of $f_{n}$. By Fatou's lemma and (54), we have

$$
\begin{aligned}
\|g f\|_{B_{p}^{s, q}} & \leq \lim _{n \rightarrow \infty} \inf \left\|g f_{n}\right\|_{B_{p}^{s, q}} \\
& \leq \lim _{n \rightarrow \infty} \inf C\|g\|_{\mathscr{C}^{\alpha}}\left\|f_{n}\right\|_{B_{p}^{s, q}} \leq C\|g\|_{\mathscr{C}^{\alpha}}\|f\|_{B_{p}^{s, q}}
\end{aligned}
$$

which gives the proof of Theorem 5 .

\section{Proof of Theorem 6}

We first prove the following technical version of Theorem 6.

Lemma 12. For any $g \in \mathscr{C}^{\alpha}, f \in \mathscr{G}(\beta, \gamma)$ when $0<\beta, \gamma<\eta$, then

$$
\|f g\|_{F_{p}^{s q q}} \leq C\|g\|_{\mathscr{F}^{\alpha}}\|f\|_{F_{p}^{s q,}}
$$

where $1<p<\infty, 1<q<\infty,-\eta<s<\eta$, and $-\alpha<s<\alpha$.
Proof. Applying the wavelet expansion, for any $g \in \mathscr{C}^{\alpha}, f \in$ $\mathscr{G}(\beta, \gamma)$ when $0<\beta, \gamma<\eta$, we have

$$
\begin{aligned}
\|f g\|_{F_{p}^{s, q}} \leq & \left\|P_{0} g P_{0}^{2}(f)\right\|_{L^{p}}+\left\|P_{0} g\left(\sum_{l=0}^{\infty} Q_{l}^{2}(f)\right)\right\|_{L^{p}} \\
& +\left\|\left\{\sum_{k=0}^{\infty}\left[\delta^{-k s}\left|Q_{k} g P_{0}^{2}(f)\right|\right]^{q}\right\}^{1 / q}\right\| \\
& +\left\|\left\{\sum_{k=0}^{\infty}\left[\delta_{L^{p}}^{-k s}\left|Q_{k} g \sum_{l=0}^{\infty} Q_{l}^{2}(f)\right|\right]^{q}\right\}^{1 / q}\right\|_{L^{p}} \\
= & : Z_{1}+Z_{2}+Z_{3}+Z_{4} .
\end{aligned}
$$

The estimate of $Z_{1}$ is the same as in the proof of Theorem 5. We only estimate $Z_{2}, Z_{3}$, and $Z_{4}$. By applying the inequality (39)-(42), the Hölder inequality and the Fefferman-stein vector-valued maximal function inequality for $1<p<\infty$, $1<q<\infty$ in [20], it follows that

$$
\begin{aligned}
& Z_{2} \leq C\|g\|_{\mathscr{C}^{\alpha}}\left\|\sum_{l=0}^{\infty} \delta^{l((\alpha \wedge \eta)+s)} \delta^{-l s} M\left(Q_{l}(f)\right)\right\|_{L^{p}} \\
& \leq C\|g\|_{\mathscr{C}^{\alpha}}\left\|\left\{\sum_{l=0}^{\infty}\left[\delta^{-l s}\left|Q_{l}(f)\right|\right]^{q}\right\}^{1 / q}\right\|_{L^{p}} \\
& \leq C\|g\|_{\mathscr{C}^{\alpha}}\|f\|_{F_{p}^{s, q}} \\
& Z_{3} \leq C\|g\|_{\mathscr{C}^{\alpha}}\left\|\left\{\sum_{k=0}^{\infty}\left[\delta^{k((\alpha \wedge \eta)-s)} M\left(P_{0}(f)\right)\right]^{q}\right\}^{1 / q}\right\|_{L^{p}} \\
& \leq C\|g\|_{\mathscr{C}^{\alpha}}\left\|P_{0} f\right\|_{L^{p}} \leq C\|g\|_{\mathscr{C}^{\alpha}}\|f\|_{F_{p}^{s, q}} ; \\
& Z_{4} \leq C\|g\|_{\mathscr{C}^{\alpha}}\left\|\left\{\sum_{k=0}^{\infty}\left[\delta^{-k s} \sum_{l=0}^{\infty} \delta^{-|k-l|(\eta \wedge \alpha)} M\left(Q_{l}(f)\right)\right]^{q}\right\}^{1 / q}\right\|_{L^{p}} \\
& \leq C\|g\|_{\mathscr{C}^{\alpha}} \|\left\{\sum_{k=0}^{\infty}\left[\sum_{l=0}^{\infty} \delta^{-(k-l) s} \delta^{|k-l|(\eta \wedge \alpha)}\right]^{q / q^{\prime}}\right. \\
& \left.\times\left[\sum_{l=0}^{\infty} \delta^{-(k-l) s} \delta^{|k-l|(\eta \wedge \alpha)}\left(\delta^{-l s} M\left(Q_{l} f\right)\right)^{q}\right]\right\}^{1 / q} \|_{L^{p}} \\
& \leq C\|g\|_{\mathscr{C}^{\alpha}}\left\|\left\{\sum_{l=0}^{\infty}\left(\delta^{-k s} M\left(D_{l} f\right)\right)^{q}\right\}^{1 / q}\right\|_{L^{p}} \\
& \leq C\|g\|_{\mathscr{C}^{\alpha}}\|f\|_{F_{p}^{s, q}}
\end{aligned}
$$

where we use the fact that $-(\eta \wedge \alpha)<s<(\eta \wedge \alpha)$. This verifies Lemma 12.

To show Theorem 6, we also need the following technical lemma. 
Lemma 13 (see [18]). For any $f \in F_{p}^{s, q}$, there exists a sequence $\left\{f_{n}\right\}_{n \in \mathbb{Z}_{+}} \in \mathscr{G}(\beta, \gamma)$ for $0<\beta, \gamma<\eta$, such that $f_{n} \rightarrow f$ in $F_{p}^{s, q}$ with $\left\|f_{n}\right\|_{F_{p}^{s, q}} \leq C\|f\|_{F_{p}^{s, q}}$, where $1<p<\infty, 1<q<\infty$, $-\eta<s<\eta$.

Suppose that $f, f_{n}$ are given as in Lemma 13 and $g \in \mathscr{C}^{\alpha}$. Note that if $|s|<\beta<\eta, 0<\gamma<\eta$, then $h \in \mathscr{S}(\beta, \gamma) \subset$ $F_{p}^{s, q} \cap F_{p,}^{-s, q^{\prime}}$. By duality given in [18] and Lemma 12 , it follows that

$$
\begin{aligned}
&\left|\left\langle g\left(f_{j}-f_{k}\right), h\right\rangle\right| \leq\left\|g\left(f_{j}-f_{k}\right)\right\|_{F_{p}^{s, q}}\|h\|_{F_{p^{\prime}}^{-s, q^{\prime}}} \\
& \leq\|g\|_{\mathscr{C}^{\alpha}}\left\|f_{j}-f_{k}\right\|_{F_{p}^{s, q}}\|h\|_{\mathscr{G}(\beta, \gamma)} \longrightarrow 0 \\
& \text { as } j, k \rightarrow \infty .
\end{aligned}
$$

The above estimate implies that $\lim _{n \rightarrow \infty}\left\langle g f_{n}, h\right\rangle$ exists and the limit is independent of the choice of $f_{n}$. Therefore, for $g \in \mathscr{C}^{\alpha}, f \in F_{p}^{s, q}$ we define

$$
\langle g f, h\rangle=\lim _{n \rightarrow \infty}\left\langle g f_{n}, h\right\rangle,
$$

where $h \in \mathscr{G}(\beta, \gamma)$ for $0<\beta, \gamma<\eta$ and $f_{n}$ is a sequence defined in Lemma 13.

We now apply Fatou's lemma and Lemma 13 to show Theorem 6.

Proof of Theorem 6. For any $g \in \mathscr{C}^{\alpha}, f \in F_{p}^{s, q}$, applying Fatou's lemma and Lemma 13 implies that

$$
\begin{aligned}
\|g f\|_{F_{p}^{s, q}} & \leq\left\|\left\{\sum_{k=0}^{\infty}\left[\left.\delta^{-k s}\right|_{n \rightarrow \infty} \lim _{k} B_{k}\left(g f_{n}\right) \mid\right]^{q}\right\}^{1 / q}\right\|_{p} \\
& \leq \liminf _{n \rightarrow \infty}\left\|g f_{n}\right\|_{F_{p}^{s, q}} \\
& \leq C \liminf _{n \rightarrow \infty}\|g\|_{\mathscr{C}^{\alpha}}\left\|f_{n}\right\|_{F_{p}^{s, q}} \\
& \leq C\|g\|_{\mathscr{C}^{\alpha}}\|f\|_{F_{p}^{s, q}}
\end{aligned}
$$

where, as before, $B_{-1}=P_{0}, B_{k}=Q_{k}$ for $k \in \mathbb{Z}_{+}$.

The proof of Theorem 6 is concluded.

\section{Conflict of Interests}

The authors declare that there is no conflict of interests regarding the publication of this paper.

\section{Acknowledgments}

The second and third authors are supported by NNSFChina (Grant no. 11171345), the Doctoral Fund of Ministry of Education of China (Grant no. 20120023110003). The second author is also supported by China Scholarship Council. The third author is also supported by NNSF-China (Grant no. 51234005).

\section{References}

[1] V. G. Mazya and T. O. Shaposhnikova, Theory of Sobolev Multipliers. With Applications to Differential and Integral Operators, vol. 337 of Grundlehren der Mathematischen Wissenschaften, Springer, Berlin, Germany, 2009.

[2] N. S. Feldman, "Pointwise multipliers from the Hardy space to the Bergman space," Illinois Journal of Mathematics, vol. 43, no. 2, pp. 211-221, 1999.

[3] H. Koch and W. Sickel, "Pointwise multipliers of Besov spaces of smoothness zero and spaces of continuous functions," Revista Matematica Iberoamericana, vol. 18, no. 3, pp. 587-626, 2002.

[4] H. Triebel, Theory of Function Spaces, Birkhauser, Basel, Switzerland, 1983.

[5] H. Triebel, Theory of Function Spaces II, Birkhauser, Basel, Switzerland, 1992.

[6] R. Coifman and G. Weiss, Analyse Harmonique Non-Commutative Sur Certains Espaces Homogeneous, vol. 242 of Lecture Notes in Mathematics, Springer, New York, NY, USA, 1971.

[7] R. Coifman and G. Weiss, "Extensions of Hardy spaces and their use in analysis," Bulletin of the American Mathematical Society, vol. 83, pp. 569-645, 1977.

[8] R. A. Macías and C. Segovia, "Lipschitz functions on spaces of homogeneous type," Advances in Mathematics, vol. 33, no. 3, pp. 257-270, 1979.

[9] G. David, J. L. Journe, and S. Semmes, "Operateurs de Calder on-Zygmund, fonctions paraaccretives et interpolation," Revista Matemática Iberoamericana, vol. 1, pp. 1-56, 1985.

[10] Y. S. Han, "Calderon-type reproducing formula and the $\mathrm{Tb}$ theorem," Revista Matemática Iberoamericana, vol. 10, pp. 5191, 1994.

[11] Y. S. Han and E. Sawyer, "Littlewood-Paley theory on spaces of homogeneous type and the classical function spaces," Memoirs of the American Mathematical Society, vol. 110, no. 530, 126 pages, 1994.

[12] Y.-S. Han, "Plancherel-pôlya type inequality on spaces of homogeneous type and its applications," Proceedings of the American Mathematical Society, vol. 126, no. 11, pp. 3315-3327, 1998.

[13] A. Nagel and E. M. Stein, "On the product theory of singualr integrals," Revista Matematica Iberoamericana, vol. 20, pp. 531561, 2004.

[14] Y. Han, "Pointwise multipliers on inhomogeneous Besov and Triebel-Lizorkin spaces in the setting of spaces of homogeneous type," Taiwanese Journal of Mathematics, vol. 17, pp. 179-206, 2013.

[15] Y. Han, "Pointwise multipliers of Besov spaces on CarnotCarathéodory spaces," Journal of Mathematical Analysis and Applications, vol. 396, pp. 578-589, 2012.

[16] Y. Han, "Pointwise multipliers of Triebel-Lizorkin spaces on Carnot-Carathéodory spaces," Journal of Function Spaces and Applications, vol. 2012, Article ID 153849, 21 pages, 2012.

[17] P. Auscher and T. Hytönen, "Orthonormal bases of regular wavelets in spaces of homogeneous type," Applied and Computational Harmonic Analysis, vol. 34, pp. 266-296, 2013.

[18] C. Chen, J. Li, and F. Liao, "Some function spaces via orthonormal bases on spaces of homogeneous type," submitted.

[19] Y. S. Han, J. Li, and L. Ward, "Product $H^{p}, C M O^{p}, V M O$ and duality via orthenormal bases on spaces of homogeneous type," preprint.

[20] C. Feferman and E. M. Stein, "Some maximal inequalities," American Journal of Mathematics, vol. 93, pp. 107-115, 1971. 


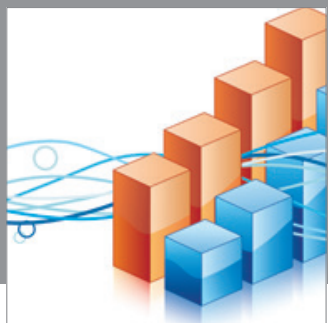

Advances in

Operations Research

mansans

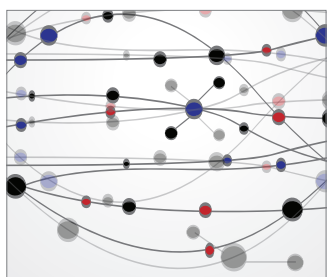

The Scientific World Journal
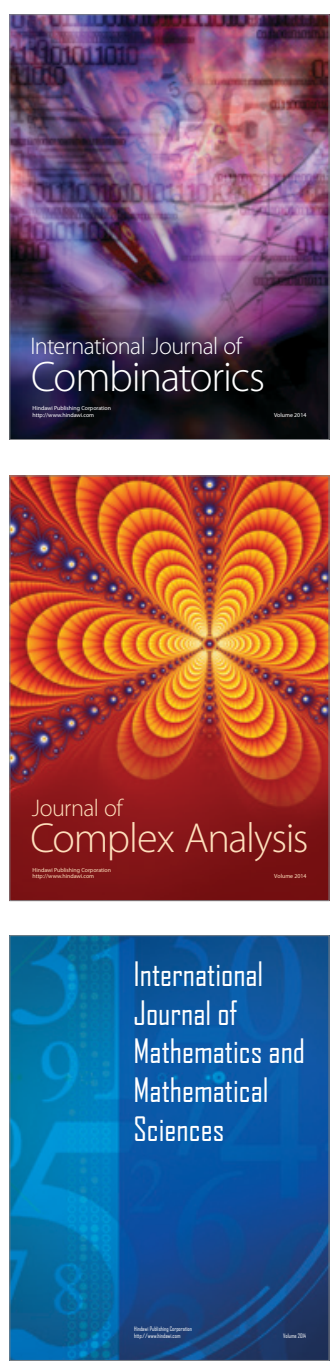
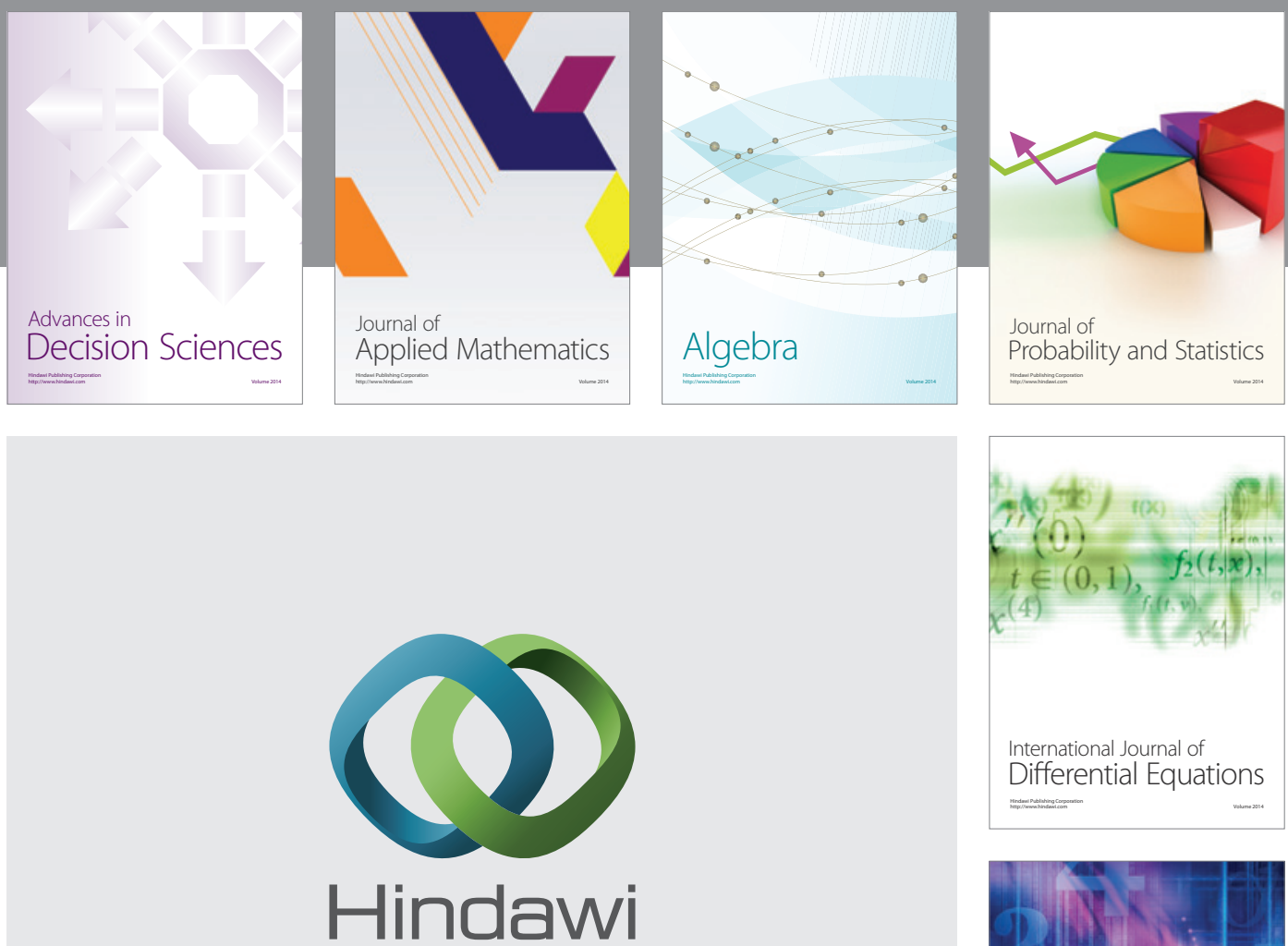

Submit your manuscripts at http://www.hindawi.com
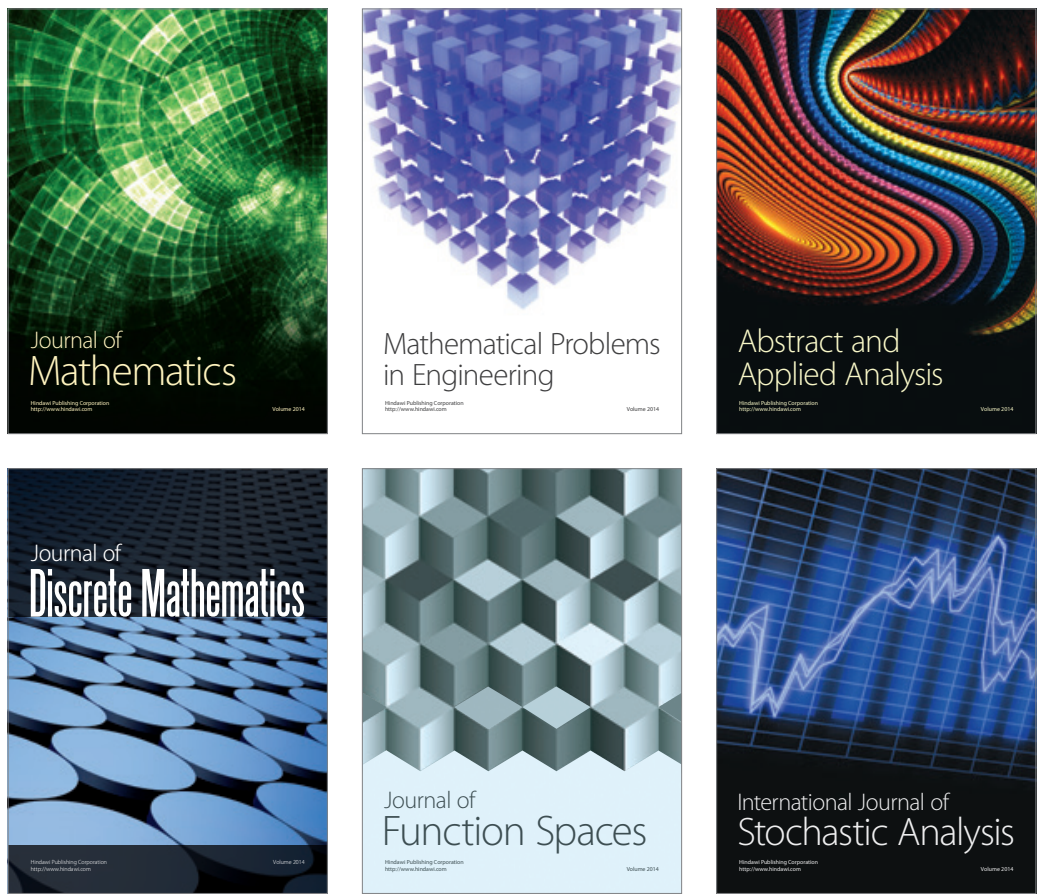

Journal of

Function Spaces

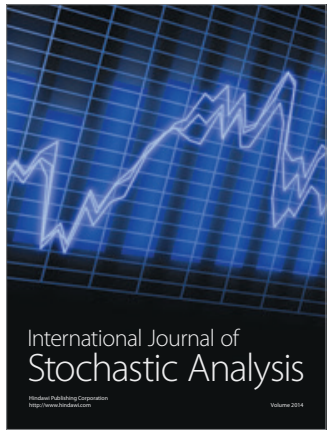

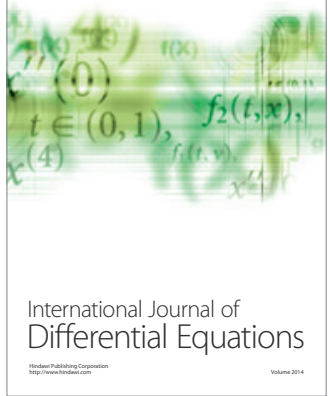
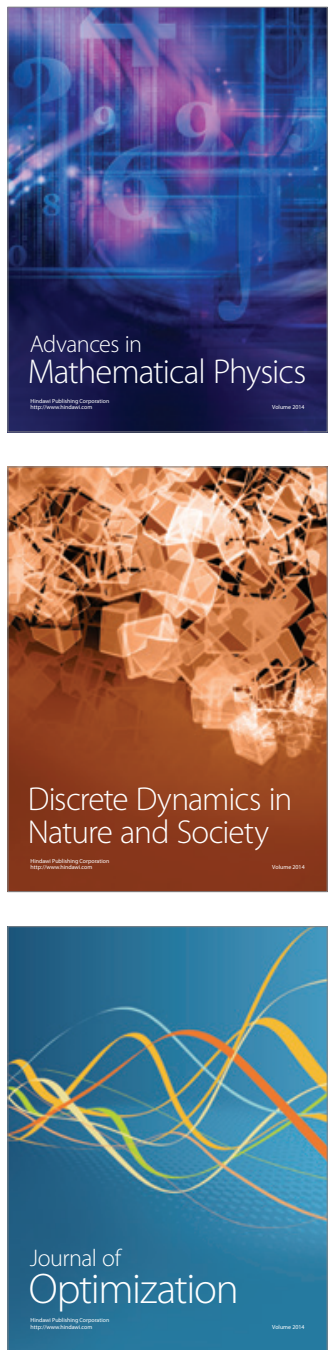